\title{
Development of an open sensorized platform in a smart agriculture context: A vineyard support system for monitoring mildew disease
}

\author{
Sergio Trilles ${ }^{\mathrm{a}}$, Joaquín Torres-Sospedra ${ }^{\mathrm{a}}$, Óscar Belmonte ${ }^{\mathrm{a}}$, F. Javier \\ Zarazaga-Soria $^{\mathrm{b}}$, Alberto González-Pérez ${ }^{\mathrm{a}}$, Joaquín Huerta ${ }^{\mathrm{a}}$ \\ ${ }^{a}$ Institute of New Imaging Technologies, Universitat Jaume I (Spain) \\ ${ }^{b}$ Aragón Institute for Engineering Research (I3A), Universidad de Zaragoza (Spain)
}

\begin{abstract}
In recent years, some official reports, to produce best products regarding quality, quantity and economic conditions, recommend that the farming sector should benefit with new tools and techniques coming from Information and Communications Technology (ICT) realm. In this way, during last decade the deployment of sensing devices has increased considerably in the field of agriculture. This fact has led to a new concept called smart agriculture, and it contemplates activities such as field monitoring, which offer support to make decisions or perform actions, such as irrigation or fertilization.

Apart from sensing devices, which use the Internet protocol to transfer data (Internet of Things), there are the so-called crop models, which are able to provide added value over the data provided by the sensors, with the aim of providing recommendations to farmers in decision-making and thus, increase the quality and quantity of their production.

In this scenario, the current work uses a low-cost sensorized platform, capable of monitoring meteorological phenomena following the Internet of Things paradigm, with the goal to apply an alert disease model on the cultivation of the vine. The edge computing paradigm is used to achieve this objective; also
\end{abstract}

Email addresses: strilles@uji.es (Sergio Trilles), jtorres@uji.es (Joaquín Torres-Sospedra), oscar.belmonte@uji.es (Óscar Belmonte), javy@unizar.es (F. Javier Zarazaga-Soria), agonzal@uji.es (Alberto González-Pérez), huerta@uji.es (Joaquín Huerta)

Preprint submitted to Journal of $E^{A} T_{E} X$ Templates

March 4, 2019 
our work follows some advances from GIScience to increase interoperability. An example of this platform has been deployed in a vineyard parcel located in the municipality of Vilafamés (Castelló, Spain).

Keywords: Wireless Sensor Networks, Internet of Things, edge computing, open sensorized platform, precision agriculture, vineyard, downy mildew, Goidanich

\section{Introduction}

The Food and Agricultural Organization (FAO) predicts that the global population will reach 8 billion people by 2025 and 9.6 billion people by 2050 [1] To keep pace, food production must increase $70 \%$ by 2050 . There are some

5 obstacles to increase food production and produce such amount of food. Some of them are climate change; restricted availability of arable land; reduced productivity growth; water necessity; the impact of urbanization over agricultural labour; high price and low availability of energy, mainly from fossil fuels; among many others. Moreover, the average age of farmers is increasing because young 10 people prefer going into the industry [2].

A report from the Intergovernmental Panel on Climate Change (IPCC) 3] enumerates all effects of climate change on agriculture. They are: 1) changes in extreme weather with severe flooding and acute water shortages areas will increase around the globe; 2) Since agriculture consumes $70 \%$ of the world's fresh water supply, water management should be an adequate and controlled process.

To mitigate these challenges, the FAO 4] recommends that all farming sectors should be equipped with new tools and techniques coming from the Information and Communications Technology (ICT). During the last decade, a new movement to implant digital technology on agriculture was born, which is known as precision agriculture [5]. It aims to optimize the yield per unit of farming land by using ICT tools and technologies [6]. The objective of precision agriculture is to achieve the best products concerning quality, quantity and 
economic conditions.

Traditionally, precision agriculture makes use of sensors to monitor environmental conditions. To attain this objective, networks of these sensors are created to cover larger areas. Precision agriculture is not only attached to deploying on-site sensors but involving many areas related to robotics, computer science, and remote sensing. An example of this is the use of smartphones, which have been used to visualize on the field the data provided by sensors and offer the possibility to apply different strategies to improve productivity [7. The works presented in [8, 9] exploit the use of advances in neural networks for weed detection and plant species identification with the purpose of optimizing the herbicide application. Finally, the review introduced in [10] showed the improvements of the spatial resolution in aerial and satellite imagery for precision agriculture since the late $80 \mathrm{~s}$, which now allows fine-grained analysis at the expense of high data storage and computational resources.

In the last decade, a new approach to connect everything (sensors or actuators), as well as to monitor and act [11] using the Internet, was implanted in every aspect of our daily lives; this process has come to be called the Internet of Things (IoT). IoT describes how in the real world, physical things are integrated into the digital world of bits and bytes [12. In general, it involves the integration of each object, such as a sensor or device that is connected via wired or wireless networks using the Internet. To connect things, the Internet ${ }_{45}$ Protocol (IP) is used, in which each device has an IP address.

The IoT devices need a mechanism to communicate with the cloud side, which is called Machine to Machine (M2M) communications. M2M will work with new developments and contributes to the future of Internet [13]. Nowadays, the future of the Internet will not only consist of connecting people but also to the approach of a digital world where, ideally, everything will be connected.

Every day farmers are producing large amounts of data that are published on the cloud, with the aim of delivering benefits concerning data access, synchronization storage and to apply some analysis, the so-called Cloud Computing (CC), to make decisions. New trends that appeared after CC move the com- 
putation towards the smart devices; the benefit is to distribute the computing between different things. This paradigm is called Edge Computing (EC) [14.

The connection between the IoT and M2M with precision agriculture have produced a new concept called "smart agriculture" following other M2M based implementations such as smart cities. One of the realms in which more great 60 adaptation of such technologies has been incorporated is the viticulture. Viticulture has historically been characterized by obtaining a high-quality product. This has been made possible by many factors: the selection of suitable soil and climatic zones, the varietal vineyards and the work of the winegrowers in managing their vineyards.

Many threats can affect quantity and quality of the wine. Concretely, there are some well-known plagues attacking vineyards, and they produce substantial losses. The vine is exposed to different types of diseases or harmful agents; these are classified into four types [15]. The first category, physiological damage or disorders in the vine that are not caused by pest and diseases, are generated by lack or excess of irrigation, extreme conditions due to meteorological factors, natural ageing, deficiencies or excesses of nutrients in the vine, lousy soil, irrigation with saline water, among many others. The second category, fungus, they can even grow in both wet and dry regions. Some examples of this disease are powdery mildew, downy mildew, and bunch rot and they can even grow well in 75 both wet and dry regions. The third category, vine pests, and insects, the most damaging are those that feed on grapes, examples of them are: Tetranychus urticae, Meloidogyne, Heterodera, Ditylenchus, Lobesia botrana, Colomerus viti, Eriophyes vitis or Phylloxera vastatrix, among others. Finally, the last category is vine diseases generated by viruses and bacteria, like grapevine degeneration, grapevine decline, leafroll complex or rugose wood complex. All these diseases impoverish the quality of the grape, and as a result, they might cause severe economic losses to farmers and people related to the wine industry.

Downy mildew is probably the most studied disease, as it produces critical 
economic losses in vineyards and, consequently, in wineries ${ }^{1}$. Within this con-

text, this paper introduces the SEnviro platform for vineyards, which integrates the downy mildew model [16 for detecting the most suitable moment to treat the downy mildew disease.

In summary, the main contributions of this work are:

1. to apply a model to alert when would be the suitable moment to treat the downy mildew disease in the vineyard context;

2. to monitor vineyard fields through a new version of the SEnviro platform following the IoT paradigm and;

3. to follow the edge computing paradigm and to mitigate the communication between ends.

The remainder of the paper is organized as follows. Section 2 presents the background of the IoT in edge computing, open hardware and smart agriculture. Section 3 presents the SEnviro Platform. Section 4 details the Goidanich model used to detect the suitable moment to apply treatment for the downy mildew disease. Section 5 shows the experimentation done to test the platform. Section

${ }_{100} 6$ reviews the related work. The paper concludes in Section 7 with conclusions and future work.

\section{Background}

This section is devoted to showing the background of the IoT, Edge Computing, and Open Hardware paradigms. Also, their relation with precision agriculture through GIS is detailed.

\subsection{Io $T$ in the edge}

Cisco's report anticipates that 50 million smart objects will be connected to the Internet by 2020 [17. This situation will cause data tsunami which refers

\footnotetext{
${ }^{1}$ See http://westernfarmpress.com/grapes/powdery-mildew-always-vineyard-concern (last accessed: April 2018)
} 
to the use of the IP to achieve connectivity between these smart objects. This trend produces streams of potential information, which challenge today's data analysis systems in a centralized server in the Cloud [18, 19, 20]. IoT movement has been successfully applied in healthcare [21, 22, 23, 24] (ambient-assisted living and telemedicine), smart home and smart metering [25, 26] (devices that enable the automation of common in-house activities), video surveillance [? ] 115 (intelligent video systems), automotive and smart mobility [27, 28] (intelligent transportation systems) and smart energy and smart grid (intelligent management of energy distribution and consumption) [29].

All these applications have boosted up the popularity of "smart things" connected to the Internet, and the networks created with them. In this way, the traditional WSNs have been updated to follow an IP protocol to apply all the benefits that it entails. The new paradigm, namely cloud computing, has been added to improve and provide more advantages over the IoT [30].

The low bandwidth lacks between the producer (smart objects) and consumer parts (cloud server) and the improved performance at both computing and consumer of smart objects, has led to a new paradigm called edge computing. The main feature of edge computing is that data can be processed locally in smart devices, rather than being sent to the cloud for being processed.

The edge computing essentially means that instead of staying and working from a centralized cloud, edge nodes work at the ends of the networks. Edge computing puts some of the transactions and resources on the edge of the cloud; instead of establishing channels for storage and use in the cloud, it reduces the need for bandwidth by not sending every bit of information through channels to the cloud and instead adds it in specific access points. By using this type of distributed strategy, we can reduce costs and increase efficiency and reduce bandwidth consumption.

Edge computing arose from the need to address three issues: 1) being able to act in real-time with the incoming data; 2) working within the limits of available bandwidth; and 3) distributing the computing between the nodes, to relive the centralized server. 
Edge computing address applications and services that do not fit the paradigm of the cloud, such as applications that require very low latency and being predictable; geospatial distributed applications; fast mobile applications; distributed control systems on a large scale.

Figure 1 details the work-flow for connecting sensor nodes to users using a cloud and edge computing infrastructure. On the one hand, this figure divides the physical and cyber worlds and the IoT part with traditional IT. The Gateway GPRS Support Node (GGSN) is used to pass the barrier between the IoT device cloud and the IT cloud [31. It is responsible for the interworking between the GPRS network and Internet. On the other hand, the figure shows where the edge computing is located. The results offered by the edge computing can be sent to the cloud or directly to the clients without using any cloud infrastructure.

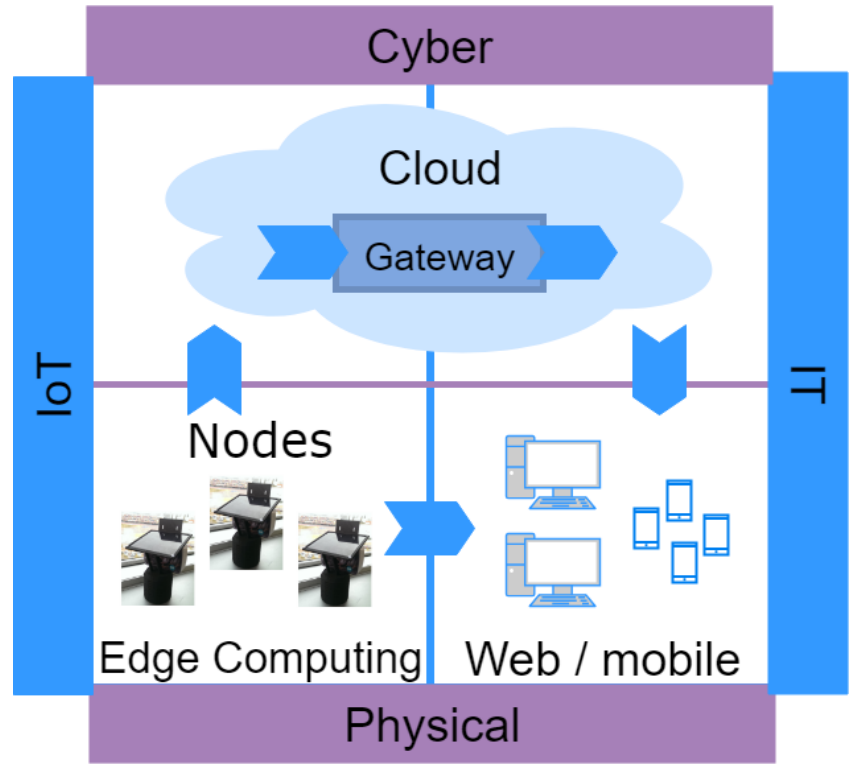

Figure 1: Representation of the cycle provided by IoT and IT, that it is divided between the physical and cyber world.

\subsection{Open-hardware to make smart objects}

Nowadays, the open movement has gained attention 32. Traditionally, this idea was applied with more acceptance over source code and provoked the 

based platform, and the most remarkable platforms are Arduind ${ }^{2}$ Raspberry $\mathrm{P}^{3}$, BeagleBon $4^{4}$ or MSP430 Launchpad ${ }^{5}$, All these platforms can be expanded easily, and used for other applications. Using these platforms different applications have been developed thanks to the acceptance of the IoT movement above

\footnotetext{
${ }^{2}$ Official Arduino Website. Available online: https://www.arduino.cc/ (accessed on 2 June 2018)

${ }^{3}$ Official RaspBerry Pi Website. Available online: http://www.raspberrypi.org (accessed on 2 June 2018).

${ }^{4}$ Official BeagleBoard Website. Available online: http://beagleboard.org (accessed on 2 June 2018).

${ }^{5}$ Official MSP430 Launchpad Website. Available online: http://www.ti.com/launchpad (accessed on 2 June 2018).
} 


\subsection{GIS role in smart agriculture}

As commented in the Section 1, one way to address these issues and increase the quality and quantity of agricultural production is by using ICT to make farms more "intelligent" and more connected through the so-called "precision agriculture" also known as "smart agriculture".

Nowadays, there is a high quantity of proprietary IT solutions to help farmers manage their farms more effectively [34. More advanced systems track geospatial zones, weather patterns and perform expert predictions [35]. These kinds of systems are called Farm Management Information Systems [36, and focus on specific tasks and use their specifications to implement the functionality provided.

Currently, this field is slowly moving to use the Internet and is starting to use some of the networking solutions to improve what they offer to the end users. However, it is widely accepted that the Internet faces some shortcomings, especially in handling vast numbers of networked devices (IoT) or stakeholders. Moreover, there is still no standardized solution to enable cohesive and straightforward interoperability among services and stakeholders.

To solve this issue, we look at GIScience. It can offer an excellent opportunity to standardize the ways of connecting different "things", offering an interoperable connection; and it should help to deploy the new smart cities. GIScience can be defined as "the discipline that uses GIS as tools to understand the world" [37. It can contribute by offering a crucial essential component for the IoT movement, which is interoperability using the use of standards.

The Open Geospatial Consortium (OGC) $)^{6}$ is a non-profit international organization which develops open standards for the global geospatial community. OGC defines standards in a wide variety of domains including environment, defense, health, agriculture, meteorology, sustainable development, and smart cities. The OGC has standardized Sensor Web Enablement (SWE) as a set of specifications related to sensors, sensor data models, and sensor web services

\footnotetext{
${ }^{6}$ See http://www.opengeospatial.org/ (last accessed: April 2018)
} 
protocol to establish the connection. With these features, it can be considered that each SEnviro Thing is a smart object. A network is formed by joining several SEnviro Things. This platform makes use of standards when publishing data. It guarantees the interoperability of the services provided by the platform.

Another important feature of this platform is that it is energetically efficient and autonomous through a battery cell, which is charged with the energy provided by a solar panel attached to it. To save as much energy as possible, we are using a sleep mode, in which during this period the micro-controller and connectivity module consume as little as possible.

\footnotetext{
${ }^{7}$ See http://ogc-iot.github.io/ogc-iot-api/ (last accessed: April 2018)
} 


\subsection{How is SEnviro made of?}

At the hardware level, the sensorized platform has been designed to be a node acting as a smart object, which provides measures of environmental variables. Each SEnviro Thing is composed of different components, which are organized into four groups depending on their function: Core, Sensors, Power Supply and

235 Communication. At the same time, the Core is formed by four parts: Microcontroller, Connectors, Clock, and Memory.

For this work, we developed an enhanced SEnviro using the LinkIt One development board. The LinkIt ONE board is an open source, high performance, Arduino footprint board for prototyping IoT devices. The board is based around ${ }^{40}$ a powerful ARM7 EJ-S processor but has onboard GSM, GPRS, Wi-Fi, Bluetooth BR/EDR/BLE, GPS, Audio codec, and SD card connector. Pin-out similar to Arduino boards, including Digital I/O, Analog I/O, PWM, I2C, SPI, UART and power supply, compatible with Grove 4-pin interface. In Section 5. we detail all components used to develop the SEnviro node for monitoring 245 vineyards.

\subsection{How does SEnviro work?}

At an operational level, SEnviro Thing can change its behaviour, updating the information about what sensor is active and the frequency at which measures are taken. The SEnviro Thing's behaviour has two stages, initial and repetitive periodic. In the initial stage, a set of procedures are executed when it is initiated, for instance, one procedure could initialize the clock using the current time. The repetitive periodic stage defines the procedures that are repeated during the SEnviro Thing's life cycle before going to sleep.

Figure 4 shows the design of the complete infrastructure with all of the system's components, and describes each of them in different layers. If we analyze SEnviro from a GIS context and specify what is happening in each of its layers, we obtain the Figure 2, which we are going to detail below. In the content layer, there are different SEnviro Things to cover an area to measure 
the phenomena by the included sensors in each sensorized platform. In this figure, we can see the two functionalities mentioned above.

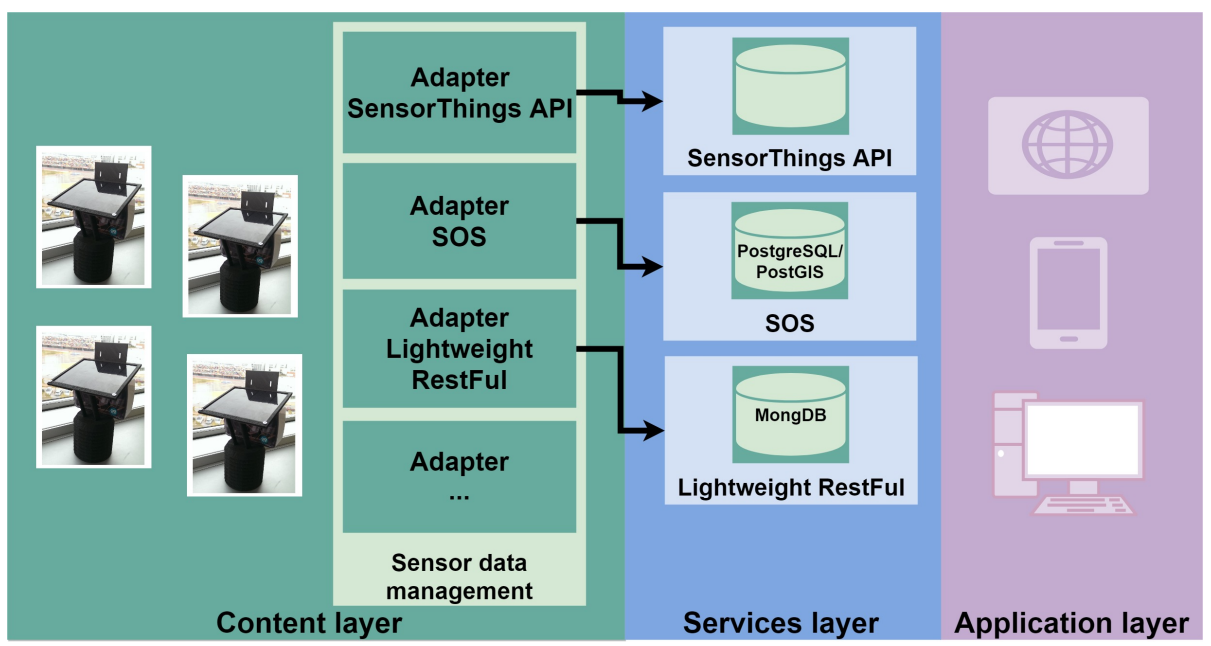

Figure 2: General overview about the SEnviro work-flow following three layers architecture.

As detailed in [39, different observations are periodically taken from the installed sensors to monitor the environment. When the SEnviro Thing takes a new observation, it invokes the Sensor Data Management (SDM) module. This component is implemented as a RESTful web service.

The SDM module is responsible for publishing observations in different services that we want to offer, such as SensorThings API, SOS or others. Thus, it offers the same content using different interfaces; depending on the context it may be more or less appropriate. Different adapters for each of these services are offered. If we want to support new services/standards, we only need to add a new adapter for the service. We use the adapter software pattern to implement this feature.

The service layer offers the services described above. One of the objectives of the SEnviro platform is to offer a standard service, to provide connectivity in an interoperable way. To meet this challenge, a candidate OGC standard, SensorThings API, has been used to offer an interoperable service to access the SEnviro platform. The most remarkable thing about this standard is that 
it breaks away from all "standard topics" [41] and offers access to restrictive devices, such as smartphones. Also, it offers the SOS interface to increase the interoperability, because this standard is widely used 42 . Finally, a lightweight RestFul service is created to connect this service in a more agile way [43].

The last layer, the application layer has been designed for different clients, mobile, web-based or desktop.

The SEnviro Thing can adapt its behavior, updating the information about which sensor is active and the frequency at which measurements are taken. At regular intervals, the platform requests a new behavior to a server using a RestFul service. Then, the SEnviro Thing will get a new behavior following the new configuration received.

The sensorized platform sends the taken observations to a server. A sensor collector service has been created to achieve this integration. Different operations are available to collect the observations measured. When one SEnviro Thing takes a new observation it invokes these operations to send data to the server using SDM module.

When the SDM receives a new observation, it connects with the different adapters to send the data for each available service. To accomplish this objective, we have followed previous work [43, 39] to connect with different OGC instances, such as SOS and candidate standard SensorThings API. Also, we provide a lighter solution for data sensors access using RestFul interface used in 43 .

To offer all these interfaces, we provide different adapters to publish the sensor data in different services or databases. To offer a SensorThings interface, we use an external server to test this API, which is offered by the SensorThings API candidate proposal. For SOS, the $52^{\circ}$ North implementation (SOS version 2.0) is used. In this case, the server is deployed using an Apache Tomcat server and sensor data are stored in a PostgreSQL/PostGIS databas $\AA^{8}$ following the documented standard installation guides. Finally, for the last interface, a

${ }^{8}$ See http://postgis.net/ (last accessed: April 2018) 
Lightweight RestFul service is implemented using JAX-RS9 Jersey ${ }^{10}$ and one MongoDB database ${ }^{11}$ is used to store the observations.

\section{Goidanich model to detect downy mildew disease}

Downy mildew is a fungal disease caused by the plasmopara viticola oomycete.

It is one of the worst diseases of vine cultivation under specific weather conditions. Downy mildew is the most studied disease, as it produces critical economic losses in vineyards and, consequently, in wineries [44]. Downy mildew is the result of the presence of a fungus in the green elements of the vines, and its life cycle is well established [44.

Its biological cycle is developed in four phases: contamination, incubation, and sporulation and propagation phase.

- Contamination phase: in spring, the oospores reach maturity and germinate as zoospores. These may be transported by rain and wind, reaching the vine green parts and colonizing them. The needed conditions for contamination are: an air temperature around $10{ }^{\circ} \mathrm{C}$, a young plant of at least $10 \mathrm{~cm}$, and a rain rate of at least $10 \mathrm{~mm} /$ day.

- Incubation phase: it takes from 4 to 21 days, depending on the temperatures and relative humidity.

- Sporulation phase: during this step, the fungus is propagated along the vine.

- Propagation phase: dissemination to nearby plants with the help of rebounding raindrops and wind. The optimal condition for ambient temperature is between 20 and 25 degrees, with the help of rain during the night.

\footnotetext{
${ }^{9}$ See http://jax-rs-spec.java.net/ (last accessed: April, 2018)

${ }^{10}$ See https://jersey.java.net/ (last accessed: April, 2018)

${ }^{11}$ See https://www.mongodb.org/ (last accessed: April, 2018)
} 

Goldanich [45] such as MILVIT [46, 47]. Some other works simulate only a particular phase of the epidemic cycle [48, 49. Moreover, some other works simulate only the first date of primary infection followed by the second cycle with quantitative outputs [50, 51]. More recently, new models are presented 52 .

Our work, unlike the ones found in the bibliography, is the pioneer in using devices of IoT for monitoring this kind of models. Although Goldanichs model has been improved over time, it is an ideal candidate for testing our platform. predictions of this type of disease.

The Goidanich model is used to follow the evolution of the downy mildew fungus [16]. It takes into account three different phenomena: relative humidity, temperature, and rainfall. The model starts when a traditional method is reached, the "rule of the three ten", which predicts that primary infections will occur once the average temperature has exceeded 10 degrees, the sprouting exceed $10 \mathrm{~cm}$ and on a day when there is at least $10 \mathrm{~mm}$ of rainfall. This rule, though simple, gives a rough idea of when to start looking for these primary infections.

To follow the evolution of the fungus, it is necessary to know the average temperature $(\mathrm{Tm})$, the average relative humidity $(\mathrm{Hm})$ and daily rainfall. Goidanich presents a daily table (Table 1) in which, for each temperature, the daily growth of the fungus is set according to whether the $\mathrm{Hm}$ is high or low. The author defines each Tm in two columns, one when the Hm is lower than $75 \%$ and the other when it is higher than $75 \%$, providing, therefore, two numerical values of daily development for each case.

When the sensor records 10 or more $\mathrm{mm}$ of rain, starting from the next day, the estimation of the daily growth is recorded, and the model is calculated by adding every day until reaching the value of 100 . At this moment, the theoretical incubation period ends, and the fungus contamination is evident 


\begin{tabular}{|c|c|c|c|c|c|c|c|c|c|c|c|}
\hline $\operatorname{Tm}(\mathrm{C})$ & & $\mathrm{Hm}<75 \%$ & $\mathrm{Hm}>75 \%$ & $\operatorname{Tm}(\mathrm{C})$ & & $\mathrm{Hm}<75 \%$ & $\mathrm{Hm}>75 \%$ & $\operatorname{Tm}(\mathrm{C})$ & & $\mathrm{Hm}<75 \%$ & $\mathrm{Hm}>75 \%$ \\
\hline \multirow[t]{4}{*}{12,} & 00 & 0,00 & 5,25 & 17 & 00 & 10 & 12,25 & 22 & 00 & 16,60 & 22,20 \\
\hline & 25 & 4,40 & 5,75 & & 25 & 10,30 & 16,60 & & 25 & 17 & 22,60 \\
\hline & 50 & 4,70 & 6,20 & & 50 & 10,50 & 14,30 & & 50 & 17,30 & 23,50 \\
\hline & 75 & 5 & 6,70 & & 75 & 10,75 & 14,75 & & 75 & 17,70 & 24,40 \\
\hline \multirow[t]{4}{*}{13} & 00 & 5,30 & 7,10 & 18 & 00 & 11,10 & 15,30 & 23 & 00 & 18,10 & 25 \\
\hline & 25 & 5,70 & 7,70 & & 25 & 11,48 & 15,20 & & 25 & 18,10 & 25 \\
\hline & 50 & 6 & 8 & & 50 & 11,70 & 16 & & 50 & 18,10 & 25 \\
\hline & 75 & 6,30 & 8,50 & & 75 & 12,10 & 16,30 & & 75 & 18,10 & 25 \\
\hline \multirow[t]{4}{*}{14} & 00 & 6,6 & 9 & 19 & 00 & 12,50 & 16,60 & 24 & 00 & 18,10 & 25 \\
\hline & 25 & 6,8 & 9,40 & & 25 & 12,90 & 17,50 & & 25 & 17,70 & 24,30 \\
\hline & 50 & 7,10 & 9,70 & & 50 & 13,40 & 18,30 & & 50 & 17,30 & 23,60 \\
\hline & 75 & 7,30 & 10,20 & & 75 & 13,70 & 19,30 & & 75 & 16,60 & 23,20 \\
\hline \multirow[t]{4}{*}{15} & 00 & 7,60 & 10,60 & 20 & 00 & 14,20 & 20 & 25 & 00 & 16,60 & 22,20 \\
\hline & 25 & 7,80 & 10,80 & & 25 & 14,50 & 20,50 & & & & \\
\hline & 50 & 8,10 & 11,10 & & 50 & 14,80 & 21 & & & & \\
\hline & 75 & 8,30 & 11,30 & & 75 & 15 & 21,51 & & & & \\
\hline \multirow[t]{4}{*}{16,} & 00 & 8,50 & 11,70 & 21, & 00 & 15,30 & 22,20 & & & & \\
\hline & 25 & 9 & 12 & & 25 & 15,70 & 22,20 & & & & \\
\hline & 50 & 9,30 & 12,50 & & 50 & 16 & 22,20 & & & & \\
\hline & 75 & 9,60 & 12,90 & & 75 & 16,30 & 22,20 & & & & \\
\hline
\end{tabular}

Table 1: Daily cumulative percentage of downy mildew incubation.

due to the appearance of stains of oil and the asexual fruiting.

The model works in that if the sum of the daily development accumulated is over $70 \%$, the risk is high, so treatment is needed as soon as possible. If a fungicide product is applied, the development of the fungus restarts and remains in suspension for a certain number of days, or until the product has been washed away by rain.

\section{Case study: SEnviro for agriculture}

This section details how we have developed the sensorized platform in a smart agricultural context.

\subsection{Building a SEnviro Thing for Smart Agriculture}

For this work, we have developed a new version of the sensorized platform presented in 39]. For the current case study, we only need three different phenomenon sensors, such as temperature, humidity sensor and rain gauge (Figure 
3). Also, for this case, the new sensorized platform uses a GPRS connection to offer easy installation and wireless connection in the field. The LinkIt One board offers an integrated GPRS module. Table 2 shows a general description. Figure 3 shows a SEnviro Thing assembled with all components used to follow the Goidanich model.

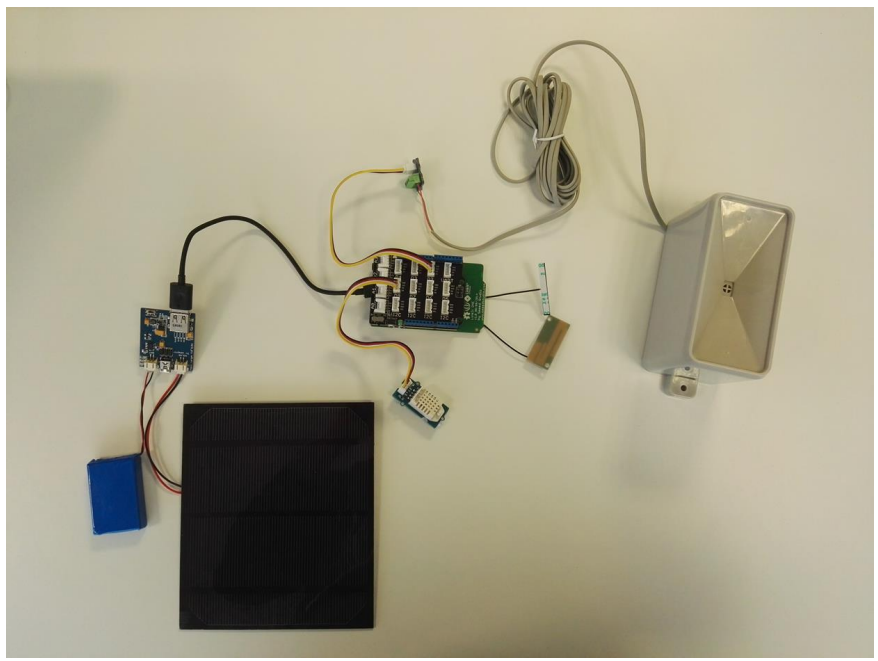

Figure 3: SEnviro Thing assembly to monitor vineyards.

To offer an energetically efficient and autonomous platform, we use a lithium battery of $2200 \mathrm{mAh}^{12}$ that supplies an output voltage of $3.7 \mathrm{~V}$ and is charged with the power generated with a $3 \mathrm{~W}$ solar pane ${ }^{13}$ that supplies an output voltage of $5.2 \mathrm{~V}$. Those two elements and the microcontroller are attached to a board (Lipo Rider V1.1 ${ }^{14}$ ) that handles the power ow between the various components.

Finally, to protect all parts of the SEnviro Thing, two boxes have been used.

\footnotetext{
12 http://www.seeedstudio.com/wiki/images/e/e5/PL805050P2200mAh_3.7V_1S1P_V_1.0. pdf (last accessed: April 2018)

${ }^{15}$ See http://www.seeedstudio.com/wiki/3W_Solar_Panel_138*160 (last accessed: April, 2018)

${ }^{14}$ See http://www.seeedstudio.com/wiki/Lipo_Rider_V1.1 (last accessed: April, 2018)
} 


\begin{tabular}{lll}
\hline \hline Category & Component & Description \\
\hline Core & Microcontroller board & Linkit One \\
& Shield Grove & Base Shield V2 \\
& MicroSD card & MicroSD card 2Gb \\
& Screw connectors (x1) & Screw Terminal for Grove \\
& Box for arduino & RETEX serie 102 \\
& Box for sensors & 3D printed box \\
\hline Communication & GPRS module & Included in LinkIt One \\
& SIM card & SIM card \\
\hline Sensors & Temperature and humid- & Grove temperature and \\
& ity sensor & humidity sensor \\
& Rainfall sensors & Weather Meters \\
\hline Power Supply & Power module & Li-po Rider \\
& Battery & Polymer Lithium Ion Bat- \\
& & tery 2200mAh 3.7V \\
& Solar panel & 3 W Solar Panel 138X160 \\
\hline \hline
\end{tabular}

Table 2: Components list included in the SEnviro Thing for smart agriculture. 
A waterproof box 15 to protect the Core, Communication and Power supply and a pagoda box to protect the sensors.

\subsection{Applying Goidanich model}

This section describes the behavior that follows the developed SEnviro node APP is a web-application in charge of integrating the sensor data provided by

\footnotetext{
${ }^{15}$ See http://www.retex.es/es/Cajas/Cajas-universales/Serie-102.axd (last accessed: April 2018)
} 


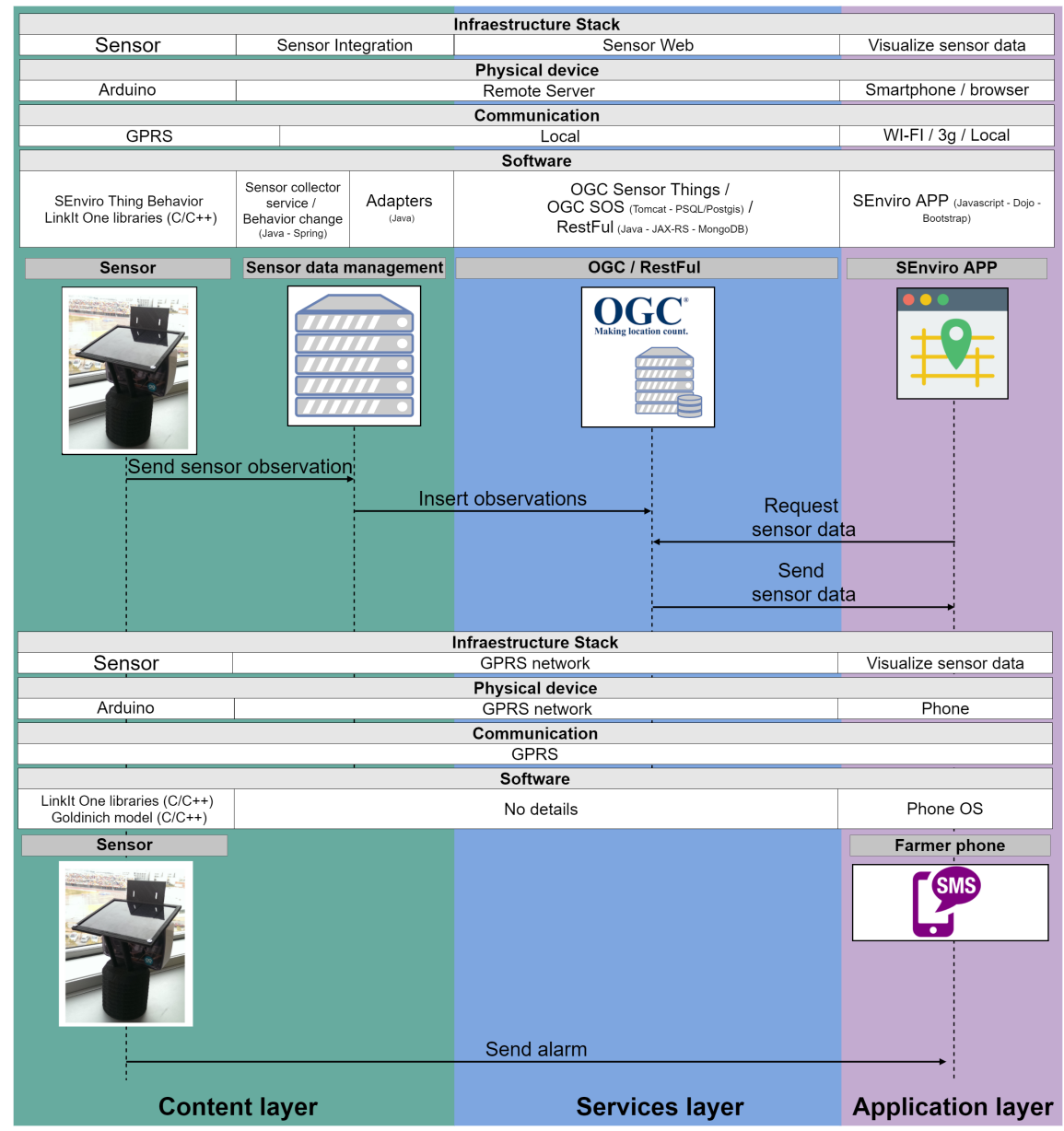

Figure 4: On top) the figure gives information about the realization of the different components of the infrastructure and the sequence diagram of an exemplary usage to send SEnviro's observations; on bottom) it gives a sequence diagram of an alarm.

the installed SEnviro Things. Furthermore, the application is built using the mobile-first approach. In this way, it can be easily tested on a mobile device.

The second functionality, which is of high importance for this work, is the possibility to apply the Goidanich model in each SEnviro node. We follow the fog computing paradigm to distribute the computation between the devices, in this case, SEnviro nodes.

To calculate the Goidanich model, we need to know the temperature and 

form will have the capability to execute a method as depicted in Figure 5 The Pseudocode 1 shows how each SEnviro Thing can calculate the Goidanich model.

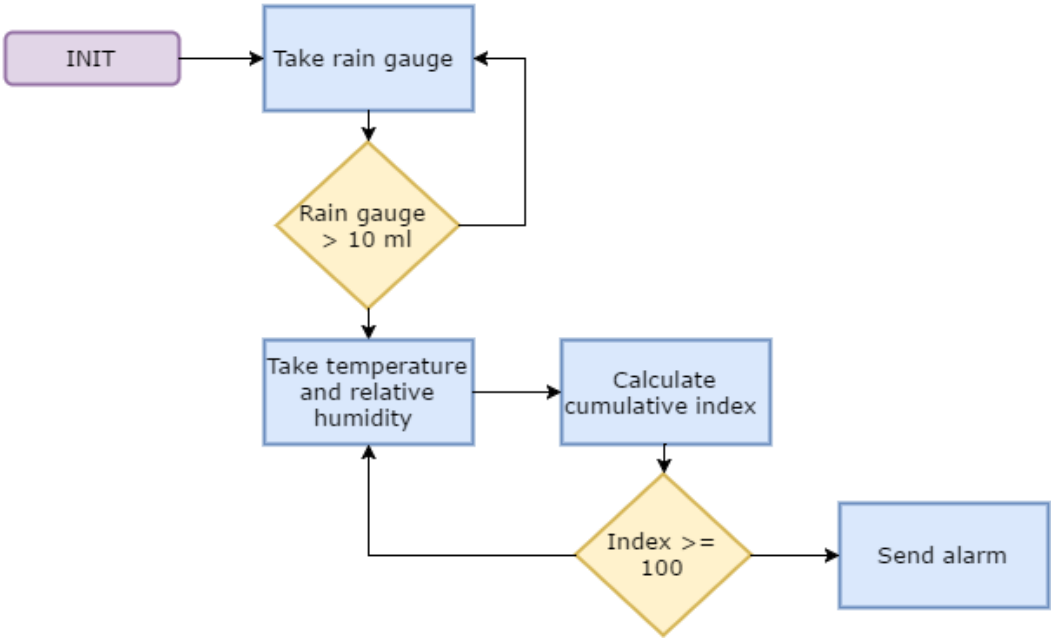

Figure 5: Goidnich model workflow.

The first step is to accumulate the rain gauge. When it starts to rain, the algorithm will calculate the rain quantitative uninterruptedly while it is raining. The accumulative value will be reset when in one-day rainfall has been recorded.

When the accumulative rainfall exceeds $10 \mathrm{~mm}$, the SEnviro starts to calculate the temperature and humidity means. To offer these means, we will add up all of the observations taken in one day and divide them by the number of observations.

These means will be used to determine the percentage of downy mildew incubation, and the selected value will be accumulated. When this value reaches 100, an alarm will be sent to the farmer to inform him/her about the downy mildew incubation level, and the specialized treatment can begin.

435

We have selected SMS-based services as the way to connect with the farmers. It is due to the high penetration of mobile phones used by the stakeholders. Also, using SMS notifications, we can guarantee that the notification will ar- 
Data: Temperature, humidity and rainfall sensors

Result: Send alarm to notify downy mildew risk acumulateGoidanich $=0$;

while true do

RainAcum = RainAcum + RainValue;

if RainAcum $>10$ then

riskMildew $=$ true (active downy mildew risk);

end

if riskMildew then

acumulateGoidanich $+=$ CalculateMildewPercent ;

if acumulateGoidanich $>100$ then

sendAlarm ;

resent all values ;

end

end

end

Algorithm 1: Applying the Goidanich model

rive at the farmer. Other mobile technologies that use Internet connection do not guarantee the notification delivery, because the receiver needs permanent

Inter-net connection. It involves farmers having a smartphone with an Internet connection data plan that will also receive the alarm.

It is important to remark the potential of the developed SEnviro node. Although this subsection has described the behavior that it followed for a particular disease, its development could be modified to follow other models using the meteorological data collected by the node. Therefore, we consider that the SEnviro node could also be useful for detecting different diseases in vineyards or other crops. 


\subsection{Results}

In a first phase, the platform has been tested in a lab environment. It was working correctly without huge losses. The sensors obtained measurements between predictable values. The rain gauge has also been tested with rain recreation, and it was working correctly. These tests were carried out both indoors and outdoors.

In a second phase, we have been deployed within a vineyard parcel located 455 in the municipality of Vilafamés (Castelló, Spain). The parcel has a 23633.4 m2. Figure 6 shows the location of each SEnviro Thing. A SEnviro node was deployed in this vineyard parcel during May 2016 (Figure 7), the month when downy mildew can begin to arise at the chosen location. As mentioned, this platform is capable of measuring three different phenomena which are temperature, humidity and the amount of rainfall that are all involved in the Goidanich's model. During this month, observations were taken every 10 minutes and were sent to the central server. Each platform can calculate the temperature and minimum humidity, in addition to calculating cumulative rainfall every day.

In this test, the platform registered correct observations. During this period, the rain gauge did not reach $10 \mathrm{~mm}$ to activate the alert, so our system does not detect downy mildew. During the 30 days that is was running almost all observations (96,9\% successful deliveries) have been measured and sent in realtime although there were some that were undetected due to the loss of the signal in the area. These lost observations were sent in following deliveries.

\section{A comparative between some similar works}

In the literature, some approaches are similar to our proposal. The following items describe the works that have been analyzed. All of them talk about IoT in the smart agriculture context, more concretely on monitoring vineyards.

- In [53], the authors propose a WSN where each node takes images from the field and internally uses image processing techniques to detect any unusual status in the leaves. 


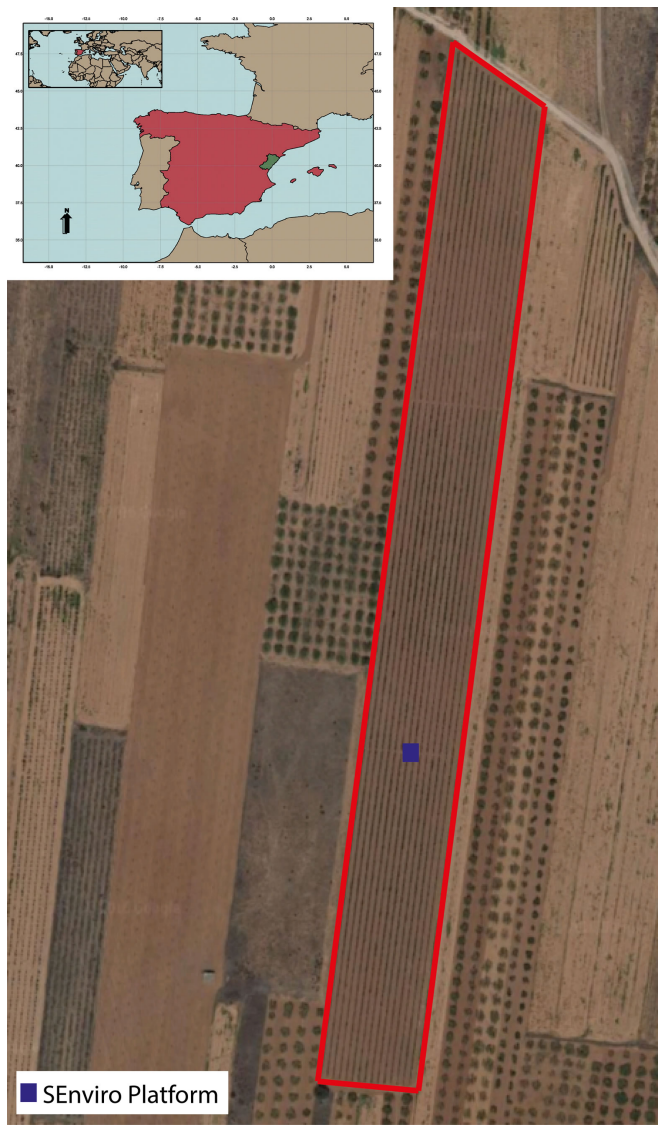

Figure 6: Location of the SEnviro Platform in a vineyard parcel located in Vilafamés (Castelló, Spain).

- The paper [54 presents a smart embedded system that will aid vineyards and which is cost-effective and flexible. They monitor ambient temperature and humidity and send an SMS with the values.

- Authors in [55] do not describe a single platform, they show a variety of sensor network configurations and applications that can address different priorities in the vineyard.

- Authors in [56] present the results from an operational sensor network. The network is based on the Berkeley mote (Mica II mote). The basic sensor board is equipped with a temperature sensor. The network con- 


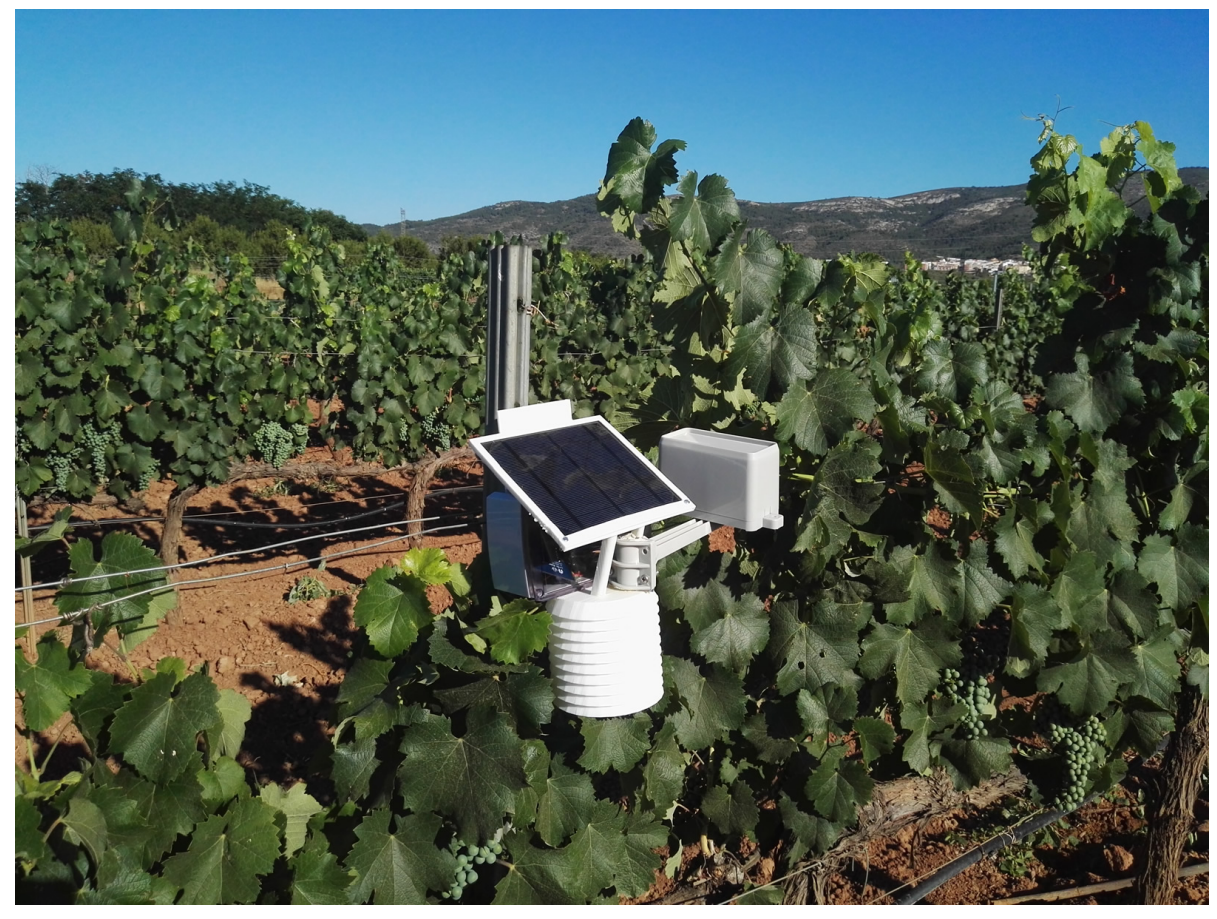

Figure 7: Picture of the SEnviro unit in the field.

sisted of sixty-five motes distributed in a grid-like pattern ten to twenty meters apart covering about two acres.

- Authors in [57] present a general sensor network designed to connect agriculture to the IoT. A customized sensor node and developed the application for agriculture on top of TinyOS 2.x are detailed. A common access interface of the sensor networks for agricultural applications was created to enable further integration of the sensor networks on the IoT.

- A sensor network built using TelosB platforms is presented in [58. They show the experience of maturated during the design and deployment of a prototypal WSN-based system for monitoring the productive cycle of high-quality wine in a Sicilian winery. They propose two networks, one for the vineyard, and another for the wine cellar. The objective is to provide some of the basic functionalities that may be extended to obtain 
complete product traceability, from the grapes to the bottle.

tween the detailed works. The following features to characterize each one have been proposed:

- Platform: it refers to the microcontroller model that the system uses.

- Real-Time: it indicates if the system works in real-time. Possible values: Yes/No.

- Connection: it refers to the wireless connections available for the system. Possible values: Wi-Fi, Bluetooth, GPRS, others.

- Phenomena: it refers to the phenomena that the system can measure. Possible values: Temp., Hum., Dust, Bar., Noise, others.

- IoT-WoT: it indicates if the system follows the IoT and WoT paradigms. Possible values: Yes/No.

- Analyze: it shows if the system offers a RESTFul interface. Possible values: Yes/No.

- Client: it indicates if the system provides a client for visualizing the sensors and observations. Possible values: Yes (what kind) /No.

- OGC standards: it shows if the system offers OGC standards. Possible values: Yes (what kind)/No.

In the following, we compare the analyzed works and our work using the characteristics listed at the beginning of this section, and the results are shown in Table 3. As indicated, most of the analyzed works use different platforms

To compare the formerly reviewed works, Table 3 shows a comparison beto build the different nodes. In our case, we use LinkIt ONE, because it has different ways to connect, GPRS, Wi-Fi or Bluetooth. It also includes useful operations to use the different features.

Like some other works analyzed, our work offers sensor data in real-time. It is possible because our SEnviro node, to monitor vineyards, is provided with GPRS 


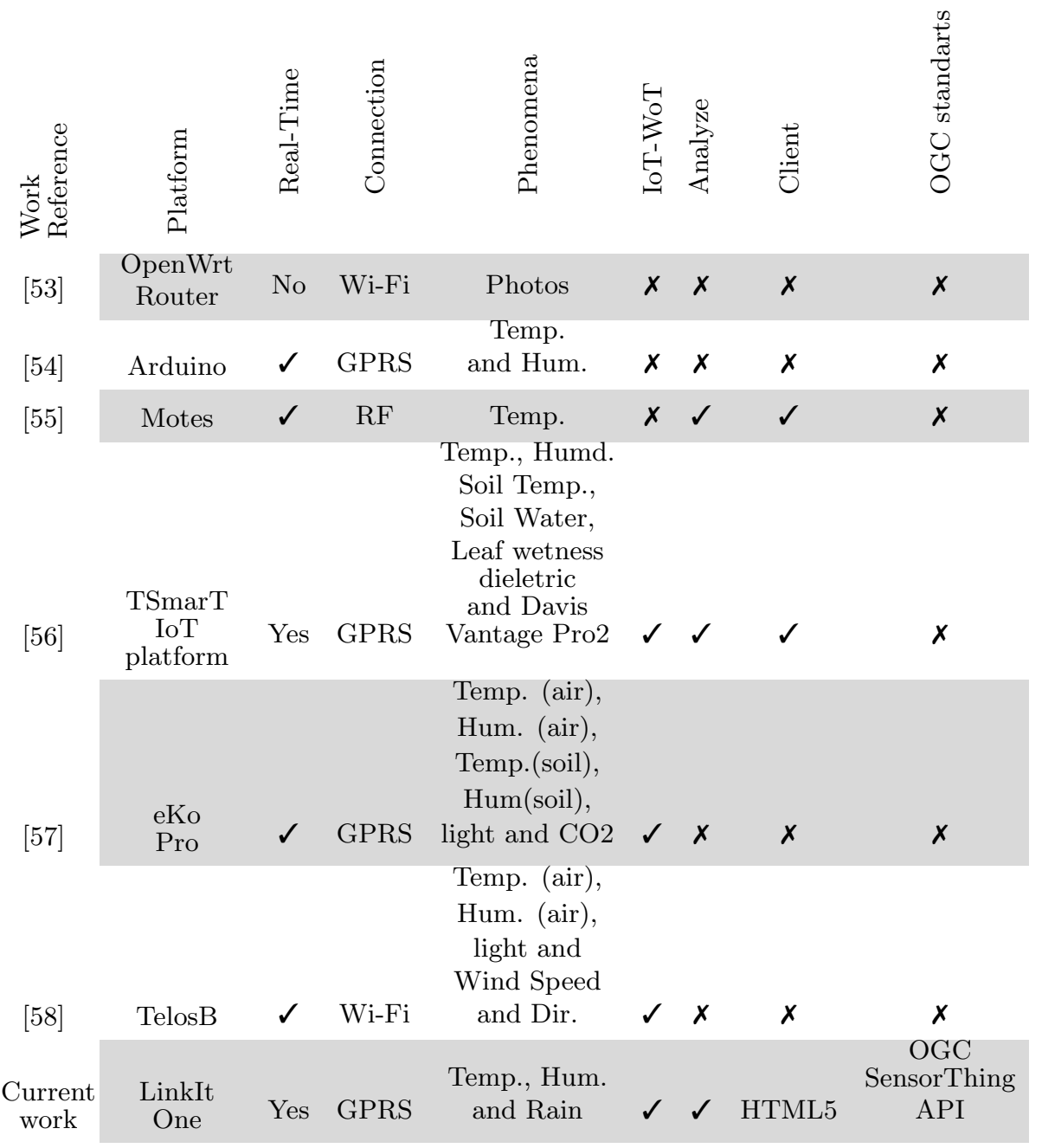

Table 3: Comparison between different sensorized platforms.

connection to the Internet. SEnviro, as we show in [39, it can include a huge variety of phenomena. For this proof of concept, to detect downy mildew disease, we adopt the nodes to offer only three phenomena: temperature, humidity and rain gauge.

Only three analyzed projects [56, 57, 58, follow the IoT and WoT paradigms. SEnviro follows these paradigms and offers a RESTFul interface. Finally, our project aims to be interoperable, and for this purpose, an OGC standard has 
been chosen, such as the OGC SensorThings API.

If you look at the works that apply some analysis over the observations from

\section{Conclusions}

540 The primary concern of this work is to apply the new improvements in the ICT area, within the smart agriculture context. In this way, the applied model to detect the suitable moment to treat downy mildew disease can help the farmer in different ways. The first improvement is the reduction of costs of chemical products. The proposed support system detects the best moment to apply the downy mildew control treatments, which are only applied when they are strictly necessary. At the same time, it reduces the chemicals in the soil. It redounds in less external elements in the environment, improving the ecological footprint of the crop. Also, it reduces costs concerning person-hours; each treatment that is not needed represents some working hours that could be used for other tasks. Consequently, the farmer does not need to visit the vineyards to know the status.

Our work applied a sensorized platform called SEnviro detailed in [39], whose objective was to monitor vineyards. For this purpose, we have designed a new version of the sensorized platform to measure different phenomena to provide follow up models to detect the best moment to treat downy mildew.

To achieve this, we propose an improved version of the SEnviro platform that follows different computer science paradigms, such as IoT or edge computing. In this way, our platform can process and apply short algorithms to compute the information at the same moment when the data are collected.

The objective of the work is not to evaluate or validate the Goldanich model since there are different works in the bibliography related to that. Our work only 
shows a single experimentation to test the presented IoT platform. It has offered good results during the month that the SEnviro platform has been installed and has correctly reported the observations to the central server, the platform had some isolated losses caused by coverage problems to send the observation in realtime. Temperatures and humidities average for the monitoring of the Goidanich model have also been calculated.

As future work, new fungal disease models in the field of viticulture could be included, such as Powdery mildew, Black rot or Botrytis. All of these diseases are based on meteorological phenomena, and they are included in the SEnviro node. New kinds of crop could be supported if the disease models are based on these meteorological phenomena. Support other crops with other phenomena require to add these sensors, in this case, more efforts are needed.

\section{Acknowledgments}

Sergio Trilles has been funded by the postdoctoral programme Vali+d (GVA; grant number APOSTD/2016/058). The project is partially funded by the Universitat Jaume I - PINV 2017 (UJI-A2017-14), the Aragon regional Government (project T59_17R) and the Spanish Government (TIN2017-88002-R).

\section{References}

[1] N. Alexandratos, J. Bruinsma, et al., World agriculture towards 2030/2050: the 2012 revision, Tech. rep., ESA Working paper (2012).

[ [2] K. Benke, B. Tomkins, Future food-production systems: vertical farming and controlled-environment agriculture, Sustainability: Science, Practice and Policy 13 (1) (2017) 13-26. arXiv:https://doi.org/10.1080/ 15487733.2017.1394054, doi:10.1080/15487733.2017.1394054 URL https : //doi.org/10.1080/15487733.2017.1394054

[3] S. Solomon, D. Qin, M. Manning, Z. Chen, M. Marquis, K. Averyt, M. Tignor, H. Miller, Contribution of working group I to the fourth assessment report of the intergovernmental panel on climate change, 2007 (2007). 
[4] E-agriculture, Using ict to enable agricultural innovation systems for smallholders (2012).

[5] A. McBratney, B. Whelan, T. Ancev, J. Bouma, Future directions of precision agriculture, Precision Agriculture 6 (1) (2005) 7-23.

[6] S. Durugkar, R. C. Poonia, Optimum utilization of natural resources for home garden using wireless sensor networks, Journal of Information and Optimization Sciences 38 (6) (2017) 1077-1085. doi:10.1080/02522667. 2017.1380391 .

[7] L. Busetto, S. Casteleyn, C. Granell, M. Pepe, M. Barbieri, M. CamposTaberner, R. Casa, F. Collivignarelli, R. Confalonieri, A. Crema, et al., Downstream services for rice crop monitoring in europe: From regional to local scale, IEEE Journal of Selected Topics in Applied Earth Observations and Remote Sensing 10 (12) (2017) 5423-5441.

[8] M. Dyrmann, H. Karstoft, H. S. Midtiby, Plant species classification using deep convolutional neural network, Biosystems Engineering 151 (2016) 72

605 -80. doi:https://doi.org/10.1016/j.biosystemseng.2016.08.024.

口 URL http://www.sciencedirect.com/science/article/pii/ S1537511016301465

q [9] J. Torres-Sospedra, P. Nebot, Two-stage procedure based on a smoothed ensembles of neural networks applied to weed detec610 tion in orange groves, Biosystems Engineering 123 (2014) 40 - 55. doi:https://doi.org/10.1016/j.biosystemseng.2014.05.005

a URL http://www.sciencedirect.com/science/article/pii/ S1537511014000713

[10] D. J. Mulla, Twenty five years of remote sensing in preci615 sion agriculture: Key advances and remaining knowledge gaps, Biosystems Engineering 114 (4) (2013) 358 - 371, special Issue: Sensing Technologies for Sustainable Agriculture. doi:https: 
//doi.org/10.1016/j.biosystemseng.2012.08.009.

URL http://www.sciencedirect.com/science/article/pii/

[11] D. INFSO, Networked enterprise \& rfid infso g. 2 micro \& nanosystems, in co-operation with the working group rfid of the etp eposs, internet of things in 2020, roadmap for the future [r] Information Society and Media, Tech. Rep.

[12] D. Uckelmann, M. Harrison, F. Michahelles, An architectural approach towards the future internet of things, Springer, 2011.

[13] J. Gubbi, R. Buyya, S. Marusic, M. Palaniswami, Internet of things (iot): A vision, architectural elements, and future directions, Future Generation Computer Systems 29 (7) (2013) 1645-1660.

${ }_{630}$ [14] F. Bonomi, R. Milito, J. Zhu, S. Addepalli, Fog computing and its role in the internet of things, in: Proceedings of the first edition of the MCC workshop on Mobile cloud computing, ACM, 2012, pp. 13-16.

[15] G. Martelli, Graft-transmissible diseases of grapevines: handbook for detection and diagnosis, Food \& Agriculture Org., 1993.

${ }_{635}^{63}$ [16] G. Goidànich, Manuale di patologia vegetale, Vol. 2, Edagricole, Bologna, 1964.

[17] J. Macaulay, L. Buckalew, G. Chung, Internet of things in logistics, DHL Trend Research 1 (1) (2015) 1-27.

[18] S. Sharma, V. Chang, U. Tim, J. Wong, S. Gadia, Cloud-based emerging services systems, International Journal of Information Management (2016) $1-12$.

[19] S. B. Bhushan, P. Reddy, D. V. Subramanian, X. Gao, Systematic survey on evolution of cloud architectures, International Journal of Autonomous and Adaptive Communications Systems 11 (1) (2018) 14-38. 

tems in the design of next-generation digital systems, Intelligent Automation \& Soft Computing 23 (3) (2017) 475-476. doi:10.1080/10798587. 2016.1220116 .

[21] B. Singh, S. Bhattacharya, C. Chowdhary, D. Jat, A review on internet of things and its applications in healthcare, Journal of Chemical and Pharmaceutical Sciences 10 (1) (2017) 447-452.

[22] V. M. Rohokale, N. R. Prasad, R. Prasad, A cooperative internet of things (iot) for rural healthcare monitoring and control, in: Wireless Communication, Vehicular Technology, Information Theory and Aerospace \& Electronic Systems Technology (Wireless VITAE), 2011 2nd International Conference on, IEEE, 2011, pp. 1-6.

[23] C. Doukas, I. Maglogiannis, Bringing iot and cloud computing towards pervasive healthcare, in: Innovative Mobile and Internet Services in Ubiquitous Computing (IMIS), 2012 Sixth International Conference on, IEEE, 2012, pp. 922-926.

[24] S. Amendola, R. Lodato, S. Manzari, C. Occhiuzzi, G. Marrocco, Rfid technology for iot-based personal healthcare in smart spaces, Internet of Things Journal, IEEE 1 (2) (2014) 144-152.

[25] M. Schneps-Schneppe, D. Namiot, A. Maximenko, D. Malov, Wired smart home: energy metering, security, and emergency issues, in: Ultra Modern Telecommunications and Control Systems and Workshops (ICUMT), 2012 4th International Congress on, IEEE, 2012, pp. 405-410.

[26] X. Li, R. Lu, X. Liang, X. Shen, J. Chen, X. Lin, Smart community: an internet of things application, Communications Magazine, IEEE 49 (11) (2011) 68-75.

[27] P. Hank, S. Müller, O. Vermesan, J. Van Den Keybus, Automotive ethernet: in-vehicle networking and smart mobility, in: Proceedings of the Conference 
on Design, Automation and Test in Europe, EDA Consortium, 2013, pp. 1735-1739.

675

[35] R. Nikkilä, I. Seilonen, K. Koskinen, Software architecture for farm management information systems in precision agriculture, Computers and electronics in agriculture 70 (2) (2010) 328-336. 
[36] S. Fountas, G. Carli, C. G. Sørensen, Z. Tsiropoulos, C. Cavalaris, A. Vatsanidou, B. Liakos, M. Canavari, J. Wiebensohn, B. Tisserye, Farm management information systems: Current situation and future perspectives, Computers and Electronics in Agriculture 115 (2015) 40-50.

[37] M. F. Goodchild, Twenty years of progress: Giscience in 2010, Journal of spatial information science 2010 (1) (2010) 3-20.

[38] J. J. Rodrigues, P. A. Neves, A survey on ip-based wireless sensor network solutions, International Journal of Communication Systems 23 (8) (2010) 963-981.

[39] S. Trilles, A. Luján, Ó. Belmonte, R. Montoliu, J. Torres-Sospedra, J. Huerta, Senviro: A sensorized platform proposal using open hardware and open standards, Sensors 15 (3) (2015) 5555-5582.

[40] D. Guinard, V. Trifa, T. Pham, O. Liechti, Towards physical mashups in the web of things, in: Networked Sensing Systems (INSS), 2009 Sixth International Conference on, IEEE, 2009, pp. 1-4.

[41] A. Tamayo, P. Viciano, C. Granell, J. Huerta, Empirical Study of Sensor Observation Services Server Instances, Vol. 1 of Lecture Notes in Geoinformation and Cartography, Springer Berlin Heidelberg, 2011, Ch. chapter 10, pp. $185-209$.

[42] M. Botts, G. Percivall, C. Reed, J. Davidson, Ogc® sensor web enablement: Overview and high level architecture, in: GeoSensor networks, Springer, 2008, pp. 175-190.

[43] S. Trilles, O. Belmonte, L. Diaz, J. Huerta, Mobile access to sensor networks by using gis standards and restful services, Sensors Journal, IEEE 14 (12) (2014) 4143-4153.

[44] G. Barrios, J. Reyes, Modelización del mildiu de la vid (2004). 
[45] C. Gessler, I. Pertot, M. Perazzolli, \&60; i\&62; plasmopara viticola\&60;/i\&62; \&58; a review of knowledge on downy mildew of grapevine and effective disease management, Phytopathologia Mediterranea 50 (1) (2011) 3-44.

[46] N. Muckensturm, C. Magnien, D. Jacquin, et al., Milvit, a new model for forecasting the risk of mildew., Phytoma (419) (1990) 34-36.

[47] C. Magnien, D. Jacquin, N. Muckensturm, P. Guillemard, Milvit: un modèle descriptif et quantitatif de la phase asexuée du mildiou de la vigne. présentation et premiers résultats de validation, EPPO Bulletin 21 (3) (1991) 451-459.

[48] N. Lalancette, M. Ellis, L. Madden, et al., Development of an infection efficiency model for plasmopara viticola on american grape based on temperature and duration of leaf wetness., Phytopathology 78 (6) (1988) 794-800.

[49] I. Gherardi, Modello a prognosi negativa per le infezioni primarie di plasmopara viticola, INFORMATORE AGRARIO 57 (18) (2001) 83-86.

[50] S. Orlandini, B. Gozzini, M. Rosa, E. Egger, P. Storchi, G. Maracchi, F. Miglietta, Plasmo: a simulation model for control of plasmopara viticola on grapevine1, EPPO Bulletin 23 (4) (1993) 619-626.

[51] P. Magarey, M. Wachtel, P. Weir, R. Seem, et al., A computer-based simulator for rational management of grapevine downy mildew (plasmopara viticola)., Plant Protection Quarterly 6 (1) (1991) 29-33.

[52] P. Blaise, R. Dietrich, M. Jermini, A new demand function for grapevine fruits in vinemild, in: V International Symposium on Computer Modelling in Fruit Research and Orchard Management 499, 1998, pp. 253-260.

[53] J. Lloret, I. Bosch, S. Sendra, A. Serrano, A wireless sensor network for vineyard monitoring that uses image processing, Sensors 11 (6) (2011) 6165-6196. 
[54] S. Zachariadis, T. H. Kaskalis, An embedded system for smart vineyard agriculture (2012).

[55] J. Burrell, T. Brooke, R. Beckwith, Vineyard computing: Sensor networks in agricultural production, Pervasive Computing, IEEE 3 (1) (2004) 38-45.

[56] R. Beckwith, D. Teibel, P. Bowen, Report from the field: results from an agricultural wireless sensor network, in: Local Computer Networks, 2004. 29th Annual IEEE International Conference on, IEEE, 2004, pp. 471-478.

[57] J. Ma, X. Zhou, S. Li, Z. Li, Connecting agriculture to the internet of things through sensor networks, in: Internet of Things (iThings/CPSCom), 2011 International Conference on and 4th International Conference on Cyber, Physical and Social Computing, IEEE, 2011, pp. 184-187.

[58] G. Anastasi, O. Farruggia, G. Lo Re, M. Ortolani, Monitoring high-quality wine production using wireless sensor networks, in: System Sciences, 2009. HICSS'09. 42nd Hawaii International Conference on, IEEE, 2009, pp. 1-7. 